\title{
Intrinsic localized modes in parametrically driven arrays of nonlinear resonators
}

\author{
Eyal Kenig, ${ }^{1}$ Boris A. Malomed, ${ }^{2}$ M. C. Cross, ${ }^{3}$ and Ron Lifshitz ${ }^{1, *}$ \\ ${ }^{1}$ Raymond and Beverly Sackler School of Physics and Astronomy, Tel Aviv University, Tel Aviv 69978, Israel \\ ${ }^{2}$ Department of Physical Electronics, School of Electrical Engineering, Tel Aviv University, Tel Aviv 69978, Israel \\ ${ }^{3}$ Condensed Matter Physics 114-36, California Institute of Technology, Pasadena, California 91125, USA \\ (Received 8 April 2009; revised manuscript received 13 August 2009; published 2 October 2009)
}

\begin{abstract}
We study intrinsic localized modes (ILMs), or solitons, in arrays of parametrically driven nonlinear resonators with application to microelectromechanical and nanoelectromechanical systems (MEMS and NEMS). The analysis is performed using an amplitude equation in the form of a nonlinear Schrödinger equation with a term corresponding to nonlinear damping (also known as a forced complex Ginzburg-Landau equation), which is derived directly from the underlying equations of motion of the coupled resonators, using the method of multiple scales. We investigate the creation, stability, and interaction of ILMs, show that they can form bound states, and that under certain conditions one ILM can split into two. Our findings are confirmed by simulations of the underlying equations of motion of the resonators, suggesting possible experimental tests of the theory.
\end{abstract}

DOI: 10.1103/PhysRevE.80.046202

\section{INTRODUCTION}

The study of collective nonlinear dynamics of coupled mechanical resonators has been regaining attention in recent years [1] thanks to advances in fabrication, transduction, and detection of microelectromechanical and nanoelectromechanical systems (MEMS and NEMS). Nonlinearity is readily observed in these systems [2-13], and is even proposed as a way to detect quantum behavior $[14,15]$. Typical MEMS and NEMS resonators are characterized by extremely high frequencies-from hundreds of $\mathrm{kHz}$ to a few $\mathrm{GHz}$ $[16,17]$ - and relatively weak dissipation, with quality factors $Q$ in the range of $10^{2}-10^{5}$. For such devices, under external driving conditions, transients die out rapidly, making it easy to acquire sufficient data to characterize the steady-state well. Because the basic physics of the individual elements is simple, and relevant parameters can readily be measured or calculated, the equations of motion describing the system can be established with confidence. This, and the fact that weak dissipation can be treated perturbatively, are a great advantage for comparison between theory and experiment.

Current technology enables the fabrication of large arrays, composed of hundreds or thousands of MEMS and NEMS devices, coupled by electric, magnetic, or elastic forces. These arrays offer new possibilities for quantitative studies of nonlinear dynamics in systems with an intermediate number of degrees of freedom-much larger than one can deal with in macroscopic experiments, yet much smaller than one confronts when considering nonlinear aspects of phonon dynamics in a crystal. Our studies of collective nonlinear dynamics of MEMS and NEMS were originally motivated by the experiment of Buks and Roukes [18], in which an array of 67 doubly-clamped micromechanical gold beams was parametrically excited by modulating the strength of an externally controlled electrostatic coupling between neighboring beams. These studies have led to a quantitative understanding of the collective response of such an array,

\footnotetext{
*ronlif@tau.ac.il
}

PACS number(s): 05.45. $-\mathrm{a}, 63.20 . P w, 62.25 .-\mathrm{g}, 85.85 .+\mathrm{j}$

providing explicit bifurcation diagrams that explain the transitions between different extended modes of the array as the strength and frequency of the external drive are varied quasistatically $[19,20]$. We have also considered more general issues such as the nonlinear competition between extended modes, or patterns, of the system-when many such patterns are simultaneously stable - as the external driving parameters are changed abruptly or ramped as a function of time [21]. Furthermore, we have investigated the synchronization that may occur in coupled arrays of nonidentical nonlinear resonators, based on the ability of nonlinear resonators to tune their frequency by changing their oscillation amplitude $[22,23]$.

Here we focus on a different type of nonlinear states, namely, intrinsic localized modes (ILMs), also known as discrete breathers or lattice solitons [24-26]. These localized states are intrinsic in the sense that they arise from the inherent nonlinearity of the resonators, rather than from extrinsically imposed disorder as in the case of Anderson localization. ILMs have been observed by Sato et al. [27-32] in driven arrays of micromechanical resonators. They have also been observed in a wide range of other physical systems including coupled arrays of Josephson junctions [33,34], coupled optical waveguides [35-37], two-dimensional nonlinear photonic crystals [38], highly nonlinear atomic lattices [39], and antiferromagnets [40,41]. Thus, the ability to perform a quantitative comparison between our theory and future experiments with large arrays of MEMS and NEMS resonators, may have consequences far beyond the framework of mechanical systems considered here.

We aim to predict the actual physical parameters, in realistic arrays of MEMS and NEMS resonators, for which ILMs can form and sustain themselves. Such predictions may have practical consequences for actual applications exploiting self-localization to focus energy, and others that may want to avoid energy focusing, for example in cases where very large oscillation amplitudes may lead to mechanical failure. Although quantitative analysis can be carried out directly in the framework of the underlying oscillator equations of motion, it is instructive to formulate the analysis in terms of an amplitude equation, as done previously for extended modes $[1,20,21]$. This allows one to display the range of stable 
ILMs on a reduced diagram, helping to describe the general qualitative behavior as physical parameters are varied. In Sec. II we describe the derivation of such a single amplitude equation from the coupled equations of motion that model an array of nonlinear resonators. This amplitude equation is obtained in the form of a parametrically driven damped nonlinear Schrödinger equation with an additional nonlinear damping term, also known as the forced complex GinzburgLandau equation. In most physical systems, the dissipation of energy is modeled by a linear damping term. However, it has been established, both theoretically $[1,19]$ and experimentally [42], that nonlinear damping is important for correct modeling of certain high- $Q$ nonlinear MEMS and NEMS resonators.

In Sec. III we argue that exact soliton solutions that exist in the absence of nonlinear damping can be continued to solve the full amplitude equation, with nonlinear damping. We derive an approximate analytical expression for these solitons, and then use it to find the exact soliton solutions numerically. A linear stability analysis of these soliton solutions follows in Sec. IV. In Sec. V we consider the dynamical formation of solitons and study the effects of nonlinear damping on the modulational instability of nonzero uniform solutions of the amplitude equation. In Sec. VI we study the interactions between pairs of solitons; and in Sec. VII demonstrate the splitting of a single soliton into two separate ones. Finally, we show in Sec. VIII that solitons of the full amplitude equation can form stable bound states. As emphasized in the concluding remarks in Sec. IX, all the phenomena demonstrated through the analysis of the amplitude equation are accurately reproduced in simulations of the underlying equations of motion of the coupled resonators, and therefore should also be reproducible in actual experiments with MEMS and NEMS arrays.

\section{DERIVATION OF THE AMPLITUDE EQUATION}

Lifshitz and Cross [19] modeled the array of coupled nonlinear resonators that was studied by Buks and Roukes [18] using the equations of motion

$$
\begin{aligned}
\ddot{u}_{n}+ & u_{n}+u_{n}^{3}-\frac{1}{2} Q^{-1}\left(\dot{u}_{n+1}-2 \dot{u}_{n}+\dot{u}_{n-1}\right) \\
& +\frac{1}{2}\left[D+H \cos \left(2 \omega_{p} t\right)\right]\left(u_{n+1}-2 u_{n}+u_{n-1}\right) \\
& -\frac{1}{2} \hat{\eta}\left[\left(u_{n+1}-u_{n}\right)^{2}\left(\dot{u}_{n+1}-\dot{u}_{n}\right)-\left(u_{n}-u_{n-1}\right)^{2}\left(\dot{u}_{n}-\dot{u}_{n-1}\right)\right] \\
= & 0
\end{aligned}
$$

where $u_{n}$ describes the deviation of the $n^{\text {th }}$ resonator from its equilibrium position, with $n=1 \ldots N$, and fixed boundary conditions $u_{0}=u_{N+1}=0$. Detailed arguments for the particular choice of terms introduced into the equations of motion are discussed in Ref. [19]. The terms include an elastic restoring force with both linear and cubic contributions (whose coefficients are both scaled to 1), a dc electrostatic nearestneighbor coupling term with a small ac component responsible for the parametric excitation (with coefficients $D$ and
$H$, respectively), and linear as well as cubic nonlinear dissipation terms. The nonlinear elastic term is positive, indicating a stiffening of the resonators with increasing displacement, which is the common situation when using doublyclamped beams. Both dissipation terms are taken in the nearest-neighbor form, which is motivated by the experimental indication that most of the dissipation originates from the electrostatic interaction between adjacent beams. Note that the electrostatic attractive force, acting between neighboring beams, decays with distance, and thus acts to soften the elastic restoring force. For this reason the sign in front of the coupling coefficient $D$ is positive, and accordingly the dispersion curve in the linear regime features a negative slope, or a negative group velocity.

In more recent implementations [13], the electric current damping has been reduced, and the parametric drive is applied piezoelectrically directly to each resonator, simplifying the equations modeling the array,

$$
\begin{aligned}
\ddot{u}_{n}+ & Q^{-1} \dot{u}_{n}+\left[1-H \cos \left(2 \omega_{p} t\right)\right] u_{n}+u_{n}^{3}+\hat{\eta} u_{n}^{2} \dot{u}_{n} \\
& -\frac{1}{2} D\left(u_{n+1}-2 u_{n}+u_{n-1}\right)=0 .
\end{aligned}
$$

The negative sign before the coupling coefficient $D$ models elastic coupling between adjacent beams, which is stronger as the separation between neighbors increases, thus acting to stiffen the resonators. Here the dispersion curve has a positive slope, or a positive group velocity. The coupling mechanism in the experimental setups in which ILMs have been observed is of this kind [27-32].

Because the quality factor $Q$ of a typical MEMS or NEMS resonator is high we follow the practice $[1,19,20]$ of using it to define a small expansion parameter $Q^{-1}=\epsilon \hat{\gamma}$, with $\epsilon \ll 1$, and $\hat{\gamma}$ of order unity. The driving amplitude is then expressed as $H=\epsilon \hat{h}$, with $\hat{h}$ of order unity, in anticipation of the fact that parametric oscillations at half the driving frequency require a driving amplitude which is of the same order as the linear damping rate [43].

An experimental protocol for producing ILMs in an array of resonators with a stiffening nonlinearity-albeit not the one we use below-is to drive the array at the highestfrequency extended mode. As the resonators are collectively oscillating at this mode, the frequency is raised further which results in an increase of the oscillation amplitude up to a point in which the extended pattern breaks into localized modes $[27,30]$. With this in mind-and concentrating on the case of elastic coupling where the highest-frequency mode $\omega=\sqrt{1+2 D}$ is the staggered mode, in which adjacent resonators oscillate out of phase-we write the displacement of the $n$th resonator as

$$
u_{n}=\epsilon^{1 / 2}\left[\hat{\psi}\left(\hat{X}_{n}, \hat{T}\right) e^{i(\omega t-\pi n)}+\text { c.c. }\right]+\epsilon^{3 / 2} u_{n}^{(1)}\left(t, \hat{T}, \hat{X}_{n}\right)+\ldots,
$$

with slow temporal and spatial variables $\hat{T}=\epsilon t$ and $\hat{X}_{n}$ $=\epsilon^{1 / 2} n$, and c.c. standing for the complex conjugate expression. We take the parametric drive frequency to be close to twice $\omega$ by setting $\omega_{p}=\omega+\epsilon \Omega / 2$, introduce a continuous spatial variable $\hat{X}$ in place of $\hat{X}_{n}$, and substitute the ansatz (3) 
into the equations of motion (2) term by term. Up to order $\epsilon^{3 / 2}$ we have

$$
\begin{aligned}
\ddot{u}_{n}=\epsilon^{1 / 2}\left[\left(-\omega^{2} \hat{\psi}+2 i \omega \epsilon \frac{\partial \hat{\psi}}{\partial \hat{T}}\right) e^{i(\omega t-\pi n)}+\text { c.c. }\right]+\epsilon^{3 / 2} \ddot{u}_{n}^{(1)}, \\
u_{n \pm 1}=-\epsilon^{1 / 2}\left[\left(\hat{\psi} \pm \epsilon^{1 / 2} \frac{\partial \hat{\psi}}{\partial \hat{X}}+\frac{\epsilon}{2} \frac{\partial^{2} \hat{\psi}}{\partial \hat{X}^{2}}\right) e^{i(\omega t-\pi n)}+\text { c.c. }\right] \\
+\epsilon^{3 / 2} u_{n \pm 1}^{(1)},
\end{aligned}
$$

$\epsilon \hat{h} \cos \left(2 \omega_{p} t\right) u_{n}=\epsilon^{3 / 2} \frac{\hat{h}}{2} \hat{\psi}^{*} e^{i \Omega \hat{T}} e^{i(\omega t+\pi n)}+O\left(e^{i 3 \omega t}\right)+$ c.c.,

$\epsilon \hat{\gamma} \dot{u}_{n}=\epsilon^{3 / 2} \hat{\gamma} i \omega \hat{\psi} e^{i(\omega t-\pi n)}+$ c.c.,

$u_{n}^{3}=\epsilon^{3 / 2} 3|\hat{\psi}|^{2} \hat{\psi} e^{i(\omega t-\pi n)}+O\left(e^{i 3 \omega t}, e^{i 3 \pi n}\right)+$ c.c.,

$u_{n}^{2} \dot{u}_{n}=\epsilon^{3 / 2} i \omega|\hat{\psi}|^{2} \hat{\psi} e^{i(\omega t-\pi n)}+O\left(e^{i 3 \omega t}, e^{i 3 \pi n}\right)+$ c.c.,

where $O\left(e^{i 3 \omega t}, e^{i 3 \pi n}\right)$ are fast oscillating terms with temporal frequency $3 \omega$ or spatial wave number $3 \pi$.

At order $\epsilon^{1 / 2}$ the equations of motion (2) are satisfied trivially. However, at order $\epsilon^{3 / 2}$, one must apply a solvability condition [44], requiring all terms proportional to $e^{i(\omega t-\pi n)}$ to vanish. It is this condition that leads to a partial differential equation (PDE) describing the slow dynamics of the amplitudes of the resonators,

$2 i \omega \frac{\partial \hat{\psi}}{\partial \hat{T}}+(3+i \omega \hat{\eta})|\hat{\psi}|^{2} \hat{\psi}+\frac{1}{2} D \frac{\partial^{2} \hat{\psi}}{\partial \hat{X}^{2}}+i \hat{\gamma} \omega \hat{\psi}-\frac{\hat{h}}{2} \hat{\psi}^{*} e^{i \Omega \hat{T}}=0$.

Note that while $e^{i(\omega t+\pi n)}=e^{i(\omega t-\pi n)}$, if we were to consider an arbitrary mode of wave number $k$ instead of $\pi$, the parametric term would have forced us to apply another solvability condition, requiring terms proportional to $e^{i(\omega t+k n)}$ to vanish. In that case an ansatz based on counter propagating waves is considered as the $O\left(\epsilon^{1 / 2}\right)$ solution for $u_{n}$ and a system of two coupled amplitude equations emerges, as shown in Ref. [20].

By means of rescaling,

$$
\begin{gathered}
\hat{\psi}=\sqrt{\frac{2 \omega \Omega}{3}} \psi, \quad \hat{X}=\sqrt{\frac{D}{2 \omega \Omega}} X, \quad \hat{T}=\frac{2}{\Omega} T, \\
\hat{h}=2 \omega \Omega h, \quad \hat{\gamma}=\Omega \gamma, \quad \hat{\eta}=\frac{3}{2 \omega} \eta,
\end{gathered}
$$

we transform Eq. (5) into a normalized form,

$$
i \frac{\partial \psi}{\partial T}=-\frac{\partial^{2} \psi}{\partial X^{2}}-i \gamma \psi-(2+i \eta)|\psi|^{2} \psi+h \psi^{*} e^{2 i T} .
$$

We then perform one final transformation $\psi \rightarrow \psi e^{i T}$ and arrive at an autonomous PDE, which is the amplitude equation that we study in the remainder of this work,

$$
i \frac{\partial \psi}{\partial T}=-\frac{\partial^{2} \psi}{\partial X^{2}}+(1-i \gamma) \psi-(2+i \eta)|\psi|^{2} \psi+h \psi^{*} .
$$

Equation (7) with $\eta=0$ is called the parametrically driven damped nonlinear Schrödinger equation (PDNLS). It models parametrically driven media in hydrodynamics [45-48] and optics [49,50], and was also used as an amplitude equation to study localized structures in arrays of coupled pendulums [51-53]. Recently, a pair of linearly coupled PDNLS equations was used to model coupled dual-core wave guides [54]. Equation (8) has the form of a forced complex GinzburgLandau equation [55] but with specific coefficients that are derived, via the scaling performed in Eqs. (3) and (6), from the underlying equations of motion (2).

We note that considering the equations of motion (1) (yet still with a negative sign before $D$ ) instead of Eq. (2) leads to the same Eq. (5) as above, but with different coefficients (a factor of 2 multiplying $\hat{h}$ and $\hat{\gamma}$, and a factor of 8 multiplying $\hat{\eta})$. Thus applying modified scaling [Eq. (6)] yields exactly the same amplitude equation [Eq. (8)].

\section{SOLITONS IN THE PRESENCE OF NONLINEAR DAMPING}

\section{A. Continuation of the PDNLS solitons to $\boldsymbol{\eta}>0$}

A remarkable feature of the amplitude equation (8) is that for $\eta=0$ it has exact time-independent solitonic solutions, as shown by Barashenkov et al. [56],

$$
\Psi_{ \pm}(X)=A_{ \pm} e^{-i \Theta \pm} \operatorname{sech}\left[A_{ \pm}\left(X-X_{0}\right)\right],
$$

where $X_{0}$ is an arbitrary position of the soliton, and

$$
A_{ \pm}^{2}=1 \pm \sqrt{h^{2}-\gamma^{2}}, \quad \cos \left(2 \Theta_{ \pm}\right)= \pm \sqrt{1-\frac{\gamma^{2}}{h^{2}}} .
$$

This pair of solitonic solutions exists for $\gamma<h$. It was shown in [56] that the $\Psi_{-}$soliton is unstable for all values of $\gamma$ and $h$, while the $\Psi_{+}$soliton is stable in a certain parameter range. A simple linear stability analysis shows that the zero solution $\psi(X)=0$, which exists for all parameter values, is stable only for $h<\sqrt{1+\gamma^{2}}$. This inequality also determines an upper stability limit for localized solutions of Eq. (8) that decay exponentially to zero on either side.

The aim of this section is to show that the PDNLS solitons $\Psi_{ \pm}$can be continued to nonzero nonlinear damping $\eta$. A similar calculation was performed by Barashenkov et al. [57] where they considered the addition of a spectral filtering term $-i c \partial^{2} \psi / \partial X^{2}$ to the PDNLS. We do so by expanding the stationary solutions of the full amplitude equation (8) in powers of $\eta$, which is assumed small, to get

$$
\psi(X)=\left(\phi_{0}+\eta \phi_{1}+\eta^{2} \phi_{2}+\cdots\right) e^{-i \Theta_{ \pm}},
$$

so that $\phi_{0}=\left|\Psi_{ \pm}\right|$. Denoting $\phi_{n}=u_{n}+i v_{n}$, with real $u_{n}$ and $v_{n}$, substituting $\psi$ into Eq. (8), and comparing powers of $\eta$ yields equations of the form

$$
L_{ \pm}\left(\begin{array}{c}
u_{n} \\
v_{n}
\end{array}\right)=\left(\begin{array}{l}
F_{n}\left(u_{0}, v_{0}, \ldots, u_{n-1}, v_{n-1}\right) \\
G_{n}\left(u_{0}, v_{0}, \ldots, u_{n-1}, v_{n-1}\right)
\end{array}\right),
$$

where 


$$
L_{ \pm}=\left(\begin{array}{cc}
-\partial_{X}^{2}-6 u_{0}^{2}+A_{ \pm}^{2} & 2 \gamma \\
0 & -\partial_{X}^{2}-2 u_{0}^{2}+A_{\mp}^{2}
\end{array}\right) .
$$

One can use Eq. (12) iteratively to find the $n$ th-order correction $\phi_{n}$, given all lower-order ones, provided that the right-hand side is orthogonal to the null subspace, or kernel, of the adjoint operator $L_{+}^{\dagger}$, if such a subspace exists. Indeed, in the relevant parameter range, $\gamma<h<\sqrt{1+\gamma^{2}}$, the adjoint operator $L_{ \pm}^{\dagger}$ has one zero eigenvalue, and the corresponding eigenvector consists of only odd functions of $X-X_{0}[57,58]$. On the other hand, one can verify that the functions $F_{n}$ and $G_{n}$, which originate from the nonlinear terms in Eq. (8), include only natural powers of $u_{0}, v_{0}, \ldots, u_{n-1}, v_{n-1}$. Therefore, and because $u_{0}=\left|\Psi_{ \pm}\right|, v_{0}=0$, and $L_{ \pm}$is parity preserving, the right-hand side of Eq. (12) consists of only even functions of $X-X_{0}$, for any $n$. This suggests that it is possible to continue the PDNLS solitons $\Psi_{ \pm}$to solve Eq. (8) up to any order in $\eta$. This can also be done in practice, by calculating $F_{n}$ and $G_{n}$ symbolically, expressing $L_{ \pm}$as a discrete matrix, and inverting it to find $u_{n}$ and $v_{n}$ in each iteration, although we do not follow this procedure here.

\section{B. Approximate analytical solitons with $\boldsymbol{\eta}>0$}

Motivated by the arguments above, we wish to construct an approximate analytical expression for the localized solution of the full amplitude equation (8), implementing the method of Barashenkov et al. [57]. To this end, we consider a function of the same form as $\Psi_{ \pm}$,

$$
\psi(X, T)=a(T) e^{-i \theta(T)} \operatorname{sech}\left[a(T)\left(X-X_{0}\right)\right],
$$

except that $a$ and $\theta$ are now time dependent. We multiply Eq. (8) by $\psi^{*}$, subtract the complex conjugate of the resulting equation and get

$$
\begin{aligned}
i \frac{\partial|\psi|^{2}}{\partial T}= & -\frac{\partial}{\partial X}\left(\frac{\partial \psi}{\partial X} \psi^{*}-\psi \frac{\partial \psi^{*}}{\partial X}\right)+h\left[\left(\psi^{*}\right)^{2}-\psi^{2}\right] \\
& -2 i \gamma|\psi|^{2}-2 i \eta|\psi|^{4} .
\end{aligned}
$$

By substituting $\psi=|\psi| e^{-i \chi}$, integrating over $X^{\prime}=X-X_{0}$, and assuming that $\psi \rightarrow 0$ and $\partial \psi / \partial X \rightarrow 0$ as $|X| \rightarrow \infty$, we obtain a spatially independent integral equation

$$
\frac{d}{d T} \int|\psi|^{2} d X^{\prime}=2 \int|\psi|^{2}[h \sin (2 \chi)-\gamma] d X^{\prime}-2 \eta \int|\psi|^{4} d X^{\prime} .
$$

Substituting the ansatz (14) into Eq. (16), we obtain the time evolution equation for $a$

$$
\frac{d a}{d T}=2 a\left[h \sin (2 \theta)-\gamma-\tilde{\eta} a^{2}\right]
$$

where $\tilde{\eta}=2 \eta / 3$. The time evolution equation for $\theta$ is derived in a similar way by multiplying Eq. (8) by $\psi^{*}$, adding the complex conjugate of the resulting equation, substituting the ansatz (14), and integrating over space to yield

$$
\frac{d \theta}{d T}=h \cos (2 \theta)+1-a^{2} .
$$

Equations (17) and (18) have the same form as the equations obtained in [57], whose fixed points are

$$
a_{ \pm}^{2}=\frac{1-\gamma \tilde{\eta} \pm \sqrt{h^{2}\left(1+\tilde{\eta}^{2}\right)-(\gamma+\tilde{\eta})^{2}}}{1+\tilde{\eta}^{2}}
$$

which has to be positive, and

$$
\begin{aligned}
& h \cos \left(2 \theta_{ \pm}\right)=a_{ \pm}^{2}-1, \\
& h \sin \left(2 \theta_{ \pm}\right)=\gamma+\tilde{\eta} a_{ \pm}^{2} .
\end{aligned}
$$

A linear analysis of these stationary points shows that $\left(a_{+}, \theta_{+}\right)$and $\left(a_{-}, \theta_{-}\right)$are a stable node and a saddle, respectively [57]. The saddle-node bifurcation point of these solutions occurs at

$$
h_{s n}(\widetilde{\eta})=\frac{\gamma+\tilde{\eta}}{\sqrt{1+\tilde{\eta}^{2}}}, \quad \text { where } \quad \tilde{\eta}=\frac{2}{3} \eta
$$

as long as $\gamma \widetilde{\eta}<1$. This is the approximate minimal driving strength required to support a localized structure in the array, in the presence of linear and nonlinear dissipation.

The approximate stable localized solution of the amplitude equation (8) is therefore given by

$$
\psi_{\text {app }}(X)=a_{+} e^{-i \theta_{+}} \operatorname{sech}\left[a_{+}\left(X-X_{0}\right)\right] .
$$

Substituting this expression into Eq. (3) yields an approximate expression for the displacements of the actual resonators in the array,

$$
\begin{aligned}
u_{n}(t)= & 2 \sqrt{\frac{2 \epsilon \omega \Omega}{3}} a_{+} \operatorname{sech}\left[a_{+}\left(\sqrt{\frac{2 \epsilon \omega \Omega}{D}} n-X_{0}\right)\right] \\
& \times \cos \left(\omega_{p} t-\pi n-\theta_{+}\right) .
\end{aligned}
$$

\section{Numerical solutions for solitons with $\boldsymbol{\eta}>\mathbf{0}$}

To obtain accurate solutions we solve the amplitude equation, as well as the underlying discrete equations of motion, numerically. The equations of motion (2) are initiated with the approximate expression (23) at a value of $h$ just above the saddle node $h_{s n}(21)$. We then perform a quasistatic upward sweep of $h$, raising $h$ in small increments and waiting for transients to decay at each step. To obtain the stationary solution of the amplitude equation we set $\partial \psi / \partial T=0$ in Eq. (8) and solve it numerically as a boundary value problem over an interval of length $L$, with boundary conditions $\psi(X$ $=0)=\psi(X=L)=0$. We use the approximate expression $\psi_{\text {app }}(X)[$ Eq. (22)] as an initial guess.

In Fig. 1 we compare the integral measure

$$
I\{f(X)\}=\frac{1}{2} \int_{-\infty}^{\infty} f^{2}(X) d X
$$

for the different localized solutions, where for the analytical solutions $f(X)=a \operatorname{sech}(a X) \cos (\theta)$ and $I=a \cos ^{2}(\theta)$, whereas for the stationary numerical solution $\psi(X)$ of the amplitude equation (8) $f(X)=\operatorname{Re} \psi(X)$, and for the numerical steadystate solution of the equations of motion (2) $f(X)$ is the appropriately scaled magnitude $\left|u_{n}\right|$ of the $n$th resonator mea- 


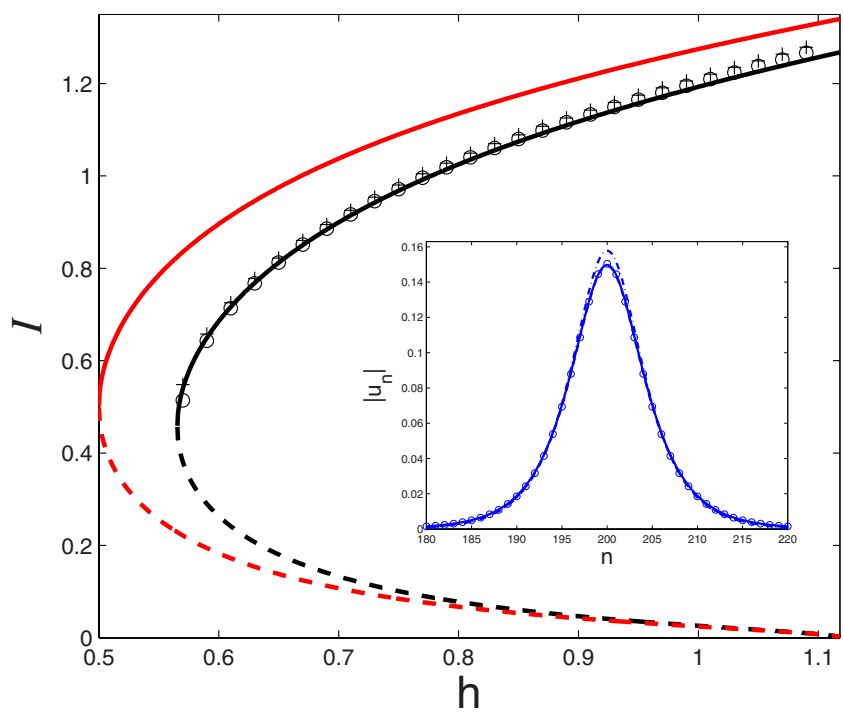

FIG. 1. (Color online) The integral measure $I\{f(X)\}$ as a function of $h$. Red outer solid and dashed lines represent the exact analytical solutions of the PDNLS equation without nonlinear damping $A_{+} \cos ^{2} \Theta_{+}$and $A_{-} \cos ^{2} \Theta_{-}$, respectively. Black inner solid and dashed lines represent the approximate analytical solutions with nonlinear damping $a_{+} \cos ^{2} \theta_{+}$and $a_{-} \cos ^{2} \theta_{-}$, respectively. These analytical lines end at the upper stability boundary $h=\sqrt{1+\gamma^{2}}$. The points designated by crosses and circles $(+$ and $\circ$ ) are taken, respectively, from the stationary numerical solution of the amplitude equation (8), and from the numerical solution of the equations of motion (2), as elaborated in the text. The inset shows the absolute value of the profile of the solution for $h=0.87$ where os are results of the numerical solution of the equations of motion (2). The solid line shows the real part of the numerical solution $\psi$ of the amplitude equation (8), scaled by a factor of $2 \sqrt{2 \epsilon \omega \Omega / 3}$. The scaled analytical approximation (22) is indistinguishable from the solid line in this plot. The dot-dashed line shows the scaled analytical solution (9) in the absence of nonlinear damping. The parameters are $\gamma=0.5, D$ $=0.25, \epsilon=0.01, \omega_{p}=1.002 \omega, \eta=0.1$, and $N=399$.

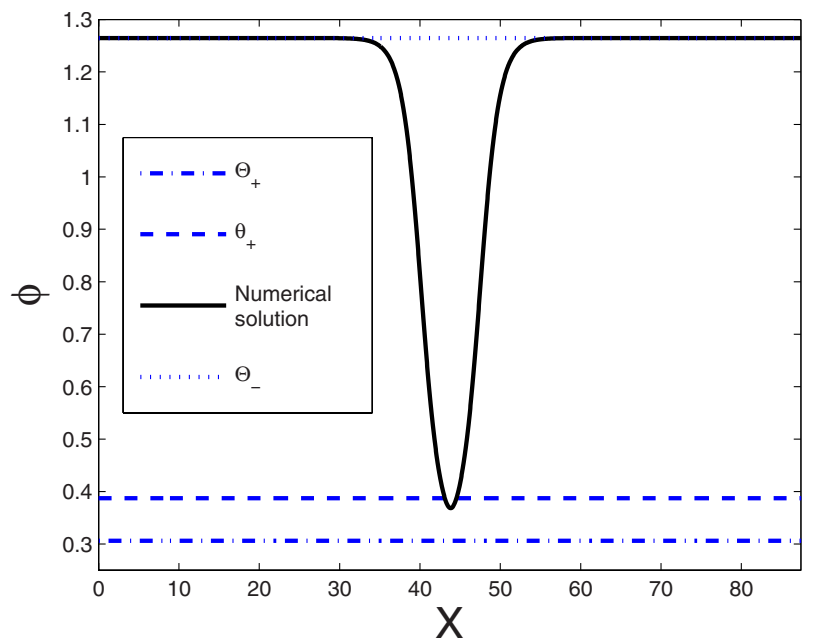

FIG. 2. (Color online) The phase of the numerical solution of the amplitude equation (8), and the analytical exact and approximate phases, as indicated in the legend. Near the peak of the soliton, the phase of the numerical solution is close to $\theta_{+}$and it asymptotes to $\Theta_{-}$as the soliton's amplitude decays to zero. Parameters are the same as in the inset of Fig. 1.

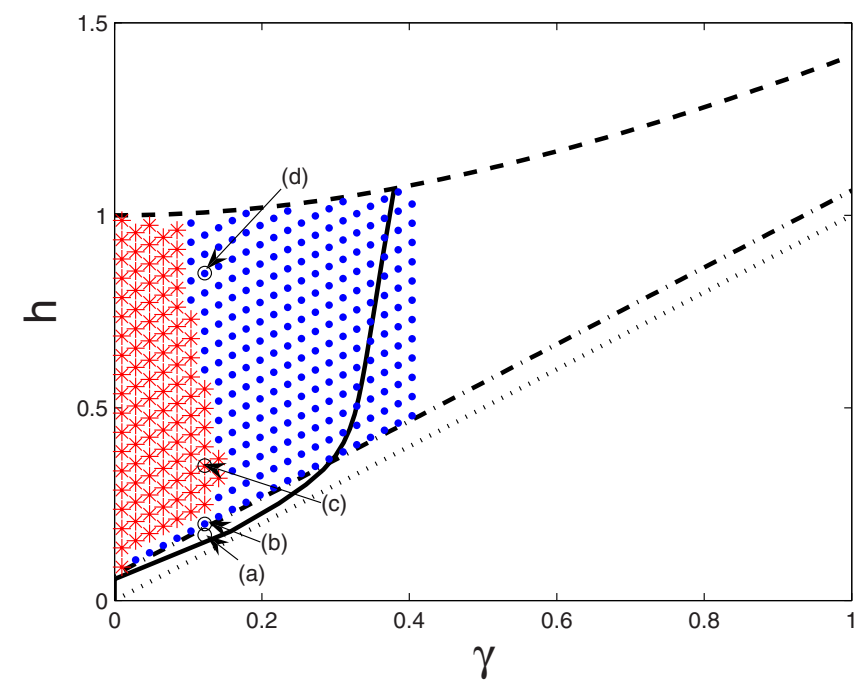

FIG. 3. (Color online) Stability diagram for localized solutions of the amplitude equation (8) in the $h$ vs $\gamma$ plane. The dotted line is the lower existence boundary for $\eta=0$, namely $h=\gamma$. The dasheddotted line is the approximate low boundary for $\eta=0.1$, given by Eq. (21). Above the solid line the $\Psi_{+}$solution of the PDNLS equation with $\eta=0$ is unstable with respect to a Hopf bifurcation [56]. The dashed line is the line $h=\sqrt{1+\gamma^{2}}$ above which the zero solution is unstable. Red asterisks $(*)$ are points for which the matrix $J^{-1} H$ has a pair of complex conjugate eigenvalues with a positive real part for $\eta=0.1$, hence the soliton solution $\psi(X)$ is unstable. Blue dots represent points for which the solution $\psi(X)$ is stable according to the linear analysis. The four black circles labeled (a)-(d) indicate parameter values corresponding to the numerical simulations of the equations of motion (2) shown in Figs. 4(a)-4(d)

sured

at times $t_{m}=2 \pi m / \omega_{p}$. Figure 1 shows good agreement between the numerical solution of the equations of motion (2), and the approximate and numerical solutions of the amplitude equation (8).

Figure 2 shows the phase of the numerical solution of the amplitude equation (8), calculated as

$$
\phi=-\arctan \left(\frac{\operatorname{Im} \psi}{\operatorname{Re} \psi}\right) .
$$

One can see that while the approximate phase $\theta_{+}$provides a good estimate for the actual phase of the solution near the peak of the soliton, the phase asymptotes to $\Theta_{-}=\pi / 2-\Theta_{+}$as the soliton's amplitude decays to zero. This is a surprising result since it might have been expected that the phase of a numerical continuation of the $\Psi_{+}$solution would tend back to $\Theta_{+}$as the amplitude drops to zero, eliminating the nonlinear damping. However, a similar situation was observed in a bound state of two $\Psi_{+}$solitons in the PDNLS equation [59].

\section{LINEAR STABILITY ANALYSIS OF SOLITONS}

Having identified an upper stability boundary $h=\sqrt{1+\gamma^{2}}$ and an approximate lower existence boundary, given by Eq. (21), we turn to examine the stability of the localized solution within these boundaries. For this purpose, we sub- 

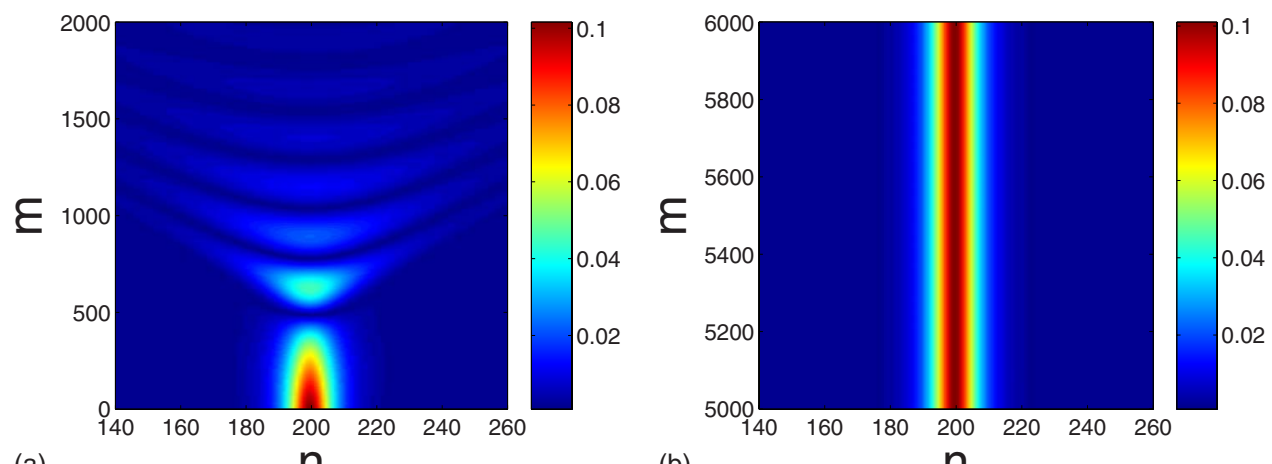

(a)

$\mathrm{n}$

(b)

n
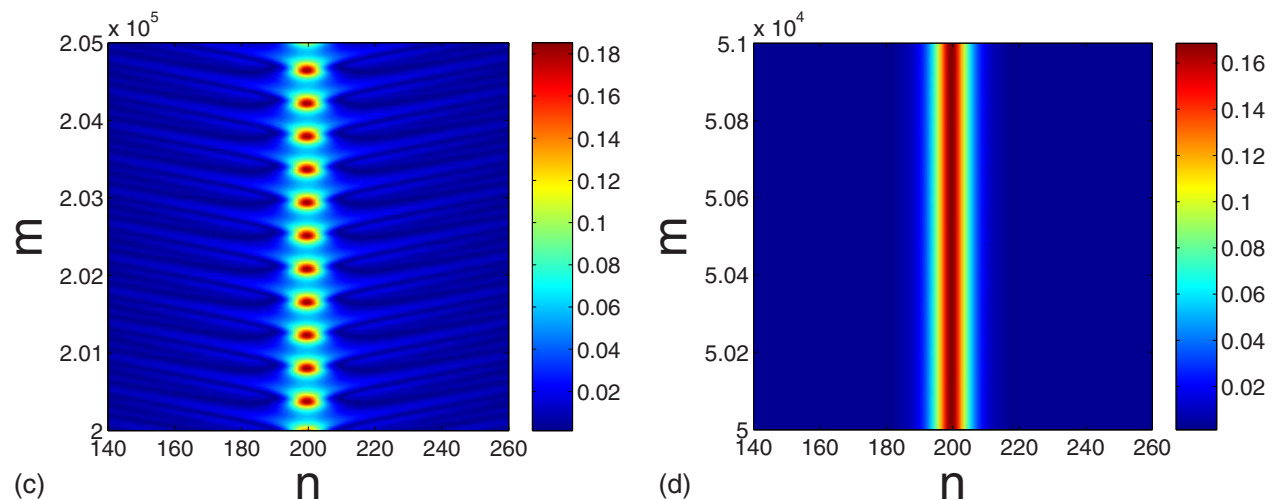

(d)

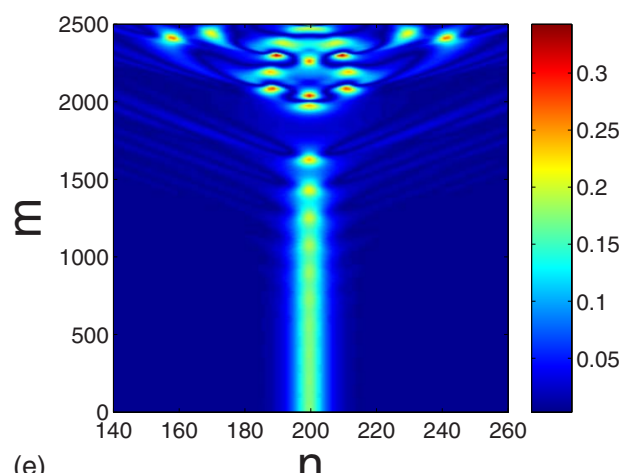

FIG. 4. (Color online) Results of the numerical solution of the equations of motion (2) for $\eta=0.1, \gamma=0.1225$, and values of $h$ labeled as (a)-(d) in Fig. 3. The solutions are plotted at times $t_{m}=2 \pi m / \omega_{p}$, with integer values $m$ shown on the vertical axis. (a) $h=0.17$ is below the approximate low boundary (21) but above the low boundary for $\eta=0$. One sees that the localized structure decays to zero. (b) $h=0.1988$ is above the approximate low boundary, where linear stability analysis predicts that the soliton is stable. (c) For $h=0.35$ the stationary soliton is unstable, and an oscillating localized solution is formed instead. In (d) $h=0.85$ and the soliton is stable again. The stability is due to nonlinear damping, without which the soliton is unstable as demonstrated in (e) where $h=0.85$ and $\eta=0$. All unspecified parameters are the same as in Fig. 1.

stitute into Eq. (8) $\psi(X, T)=\psi(X)+\delta \psi(X, T)$, where $\psi(X)$ could be any steady-state solution of the equation-in this case the stationary localized solution, which is obtained numerically - and $\delta \psi(X, T)$ is a small perturbation. We linearize in $\delta \psi(X, T)$, substituting $\psi=R+i I$ and $\delta \psi=U+i V$ with real $R, I, U$, and $V$, and obtain the equation

$$
J \frac{\partial}{\partial T}\left(\begin{array}{l}
U \\
V
\end{array}\right)=H\left(\begin{array}{l}
U \\
V
\end{array}\right),
$$

where

$$
J=\left(\begin{array}{cc}
0 & -1 \\
1 & 0
\end{array}\right), \quad H=\left(\begin{array}{ll}
H_{11} & H_{12} \\
H_{21} & H_{22}
\end{array}\right),
$$

$$
\begin{aligned}
& H_{11}=-\partial_{X}^{2}-6 R^{2}-2 I^{2}+1+h+2 \eta R I, \\
& H_{12}=\eta\left(R^{2}+3 I^{2}\right)-4 R I+\gamma, \\
& H_{21}=-\eta\left(3 R^{2}+I^{2}\right)-4 R I-\gamma, \\
& H_{22}=-\partial_{X}^{2}-6 I^{2}-2 R^{2}+1-h-2 \eta R I .
\end{aligned}
$$

By expressing the small perturbations as $U(X, T)$ $=\operatorname{Re}\left[u(X) e^{\lambda T}\right]$ and $V(X, T)=\operatorname{Re}\left[v(X) e^{\lambda T}\right]$, where $\lambda, u$, and $v$ are complex, we arrive at the eigenvalue problem 

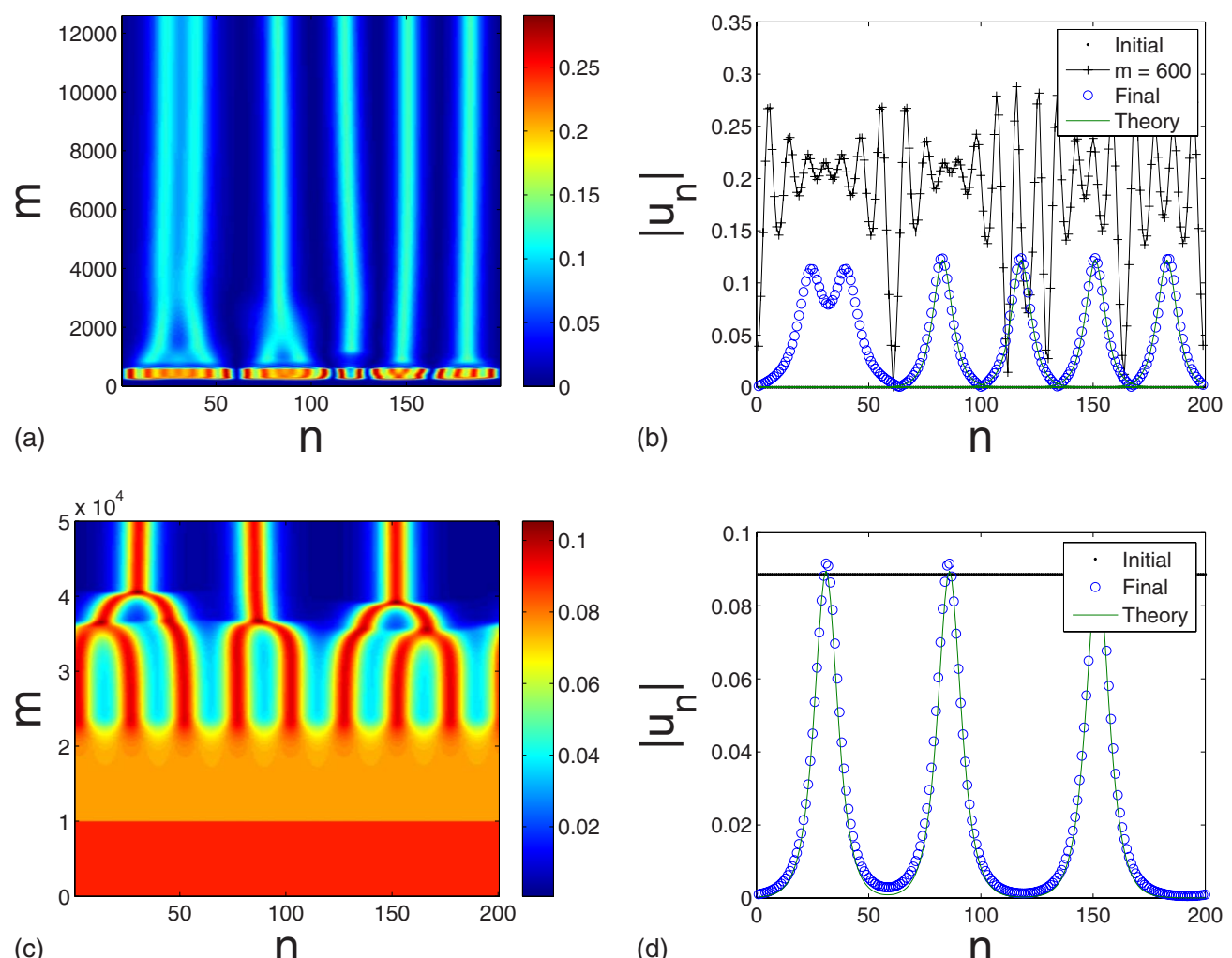

(c)

n

(d)

FIG. 5. (Color online) Numerical simulation of the coupled equations of motion (2) showing the dynamical creation of solitons. Linear damping is set to $\gamma=1$ and nonlinear damping to $\eta=0.3$. All other parameters are the same as in Fig. 1 . Plotted are the absolute values of the displacements of the resonators, which alternate between positive and negative values. Left panels show the complete time evolution, with $m$ counting the number of drive periods. Right panels show the initial (black dots) and final (blue circles) states along with the analytical form of the solitons (green solid line), using only their central positions $X_{0}$ as fitting parameters. Top panels: A simulation of 199 resonators with fixed boundary conditions is initiated with random noise and a drive amplitude of $h=5$, which is above the upper stability limit, $h=\sqrt{1+\gamma^{2}}=\sqrt{2}$, for both the zero-state and the solitons. At time $m=600$ drive periods, after some nonzero transient (black $+\mathrm{s}$ in the right panel) has developed, the drive amplitude is lowered to $h=1.35<\sqrt{2}$, yielding stable solitons. Bottom panels: A simulation of 200 resonators with periodic boundary conditions is initiated with the uniform nonzero solution and a drive amplitude of $h=1.3$, which is above the stability threshold (36), $h_{t h} \simeq 1.26$, for this state. After $m=10000$ drive periods during which the uniform state remains stable, the drive amplitude is lowered to $h=1.2<h_{t h}$, yielding stable solitons.

$$
\lambda J\left(\begin{array}{l}
u \\
v
\end{array}\right)=H\left(\begin{array}{l}
u \\
v
\end{array}\right) .
$$

When $\psi(X)$ is obtained numerically, the eigenvalues of the matrix $J^{-1} H$, describing the growth of perturbations $\delta \psi(X, T)$, are found by performing a spatial discretization of Eq. (29) and diagonalizing $J^{-1} H$ numerically (see [60] for details). The stability diagram of both the analytical solution $\Psi_{+}$for $\eta=0[56]$ and the numerical solution $\psi(X)$ for $\eta=0.1$ are displayed in Fig. 3 . These results are verified at a few points by numerical integration of the equations of motion (2), as shown in Fig. 4.

Figure 3 highlights the effects of nonlinear damping on localized solutions. The first effect is to raise the lower existence boundary. This is explained by the fact that the additional energy lost through nonlinear damping has to be compensated by an increase in the strength of the parametric drive, as predicted by the approximate expression (21). The second effect is that nonlinear damping increases the area in the $(h, \gamma)$ parameter space where solitons are stable (blue dots). In particular, the shape of the unstable

region for $\eta>0$ (red asterisks) becomes qualitatively different. There are values of $\gamma$ for which an increase in the drive amplitude $h$ initially induces an instability of the soliton, while upon further increase of $h$ the soliton regains its stability. This can be explained by noting that the amplitude of the soliton-given approximately by Eq. (19)_increases as $h$ becomes larger, thereby enhancing the effect of nonlinear damping. This increase of damping exerts a similar stabilizing effect as that of increasing $\gamma$ in the absence of nonlinear damping.

The effect of regained stability with the increase of $h$ does not occur in the absence of nonlinear damping, as the $\Psi_{+}$ soliton is unstable for all parameter values above the solid black line in Fig. 3 [56]. Different solutions of the PDNLS equation above this instability threshold were found by Bondila et al. [61] to be localized solutions that oscillate in time with different periods and chaotic solutions, in addition to the zero solution. As we increase the nonlinear damping coefficient $\eta$, the region in $(h, \gamma)$ space, in which the single soliton becomes unstable against these alternative solutions, shrinks in size. 


\section{DYNAMICAL FORMATION OF SOLITONS}

\section{A. Self-trapping of solitons}

It is not obvious how dynamically to form solitons starting with a motionless array of resonators, as one needs to take the system sufficiently far from the basin of attraction of the zero solution $\psi(X)=0$, which is also stable whenever solitons are stable. The most direct procedure for avoiding the zero solution, starting from weak random noise, is to drive the system with $h>\sqrt{1+\gamma^{2}}$, so neither the zero solution nor the soliton solutions are stable. As a consequence, a nonzero pattern develops. Stable solitons can then be formed by lowering the drive amplitude to a value $h<\sqrt{1+\gamma^{2}}$ for which the zero solution and the soliton solutions are both stable, if the nonzero pattern that was obtained is outside the basin of attraction of the zero solution.

This simple procedure-which could be implemented experimentally in a straightforward manner-is demonstrated in the top panels of Fig. 5, showing a numerical simulation of the equations of motion (2) with fixed boundary conditions, using $N=199$ resonators. One can see that the initial transient that forms becomes unstable upon lowering the drive amplitude, giving rise to the formation of a number of solitons. Note that before reaching steady state a pair of solitons merges into one, and another pair attracts and forms a bound state. Both of these effects are studied below. The emerging isolated solitons agree well with the approximate analytical form (23), determined earlier, with only their central positions $X_{0}$ used as fitting parameters.

\section{B. Modulational instability of uniform states}

A more controlled procedure for generating solitons would be to initiate the array in a particular nonzero state and drive it outside its known stability boundaries. This has been considered in the past in systems without nonlinear damping, using the nonzero uniform solution of the PDNLS $[26,57,62,63]$. However, it is known for systems with $\eta=0$ that the uniform solution is always unstable against weak modulations and so may be difficult to access dynamically. We wish to examine here whether the nonzero uniform solution may be stabilized with the help of nonlinear damping $(\eta \neq 0)$, thereby making it accessible dynamically and possibly opening an additional experimental route to the formation of solitons.

Indeed, the amplitude equation (8) admits a pair of nonzero spatially uniform solutions of the form

$$
\bar{\psi}_{ \pm}=\bar{a}_{ \pm} e^{-i \bar{\theta}_{ \pm}}
$$

Substituted into the perturbative expansion (3), this yields an oscillation of the array in its staggered mode with wave number $\pi$, about which we initially expanded our solution. If we impose fixed boundary conditions the staggered mode will be modified near the boundaries to accommodate these conditions, but would otherwise remain unchanged in the bulk of the system.

Letting $\bar{\eta}=\eta / 2$ and substituting the uniform solution (30) into the amplitude equation (8) yields

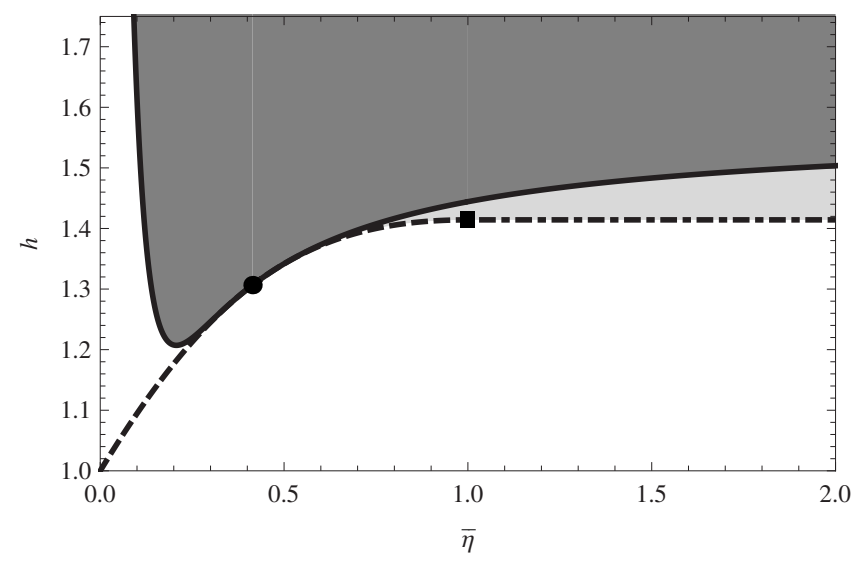

FIG. 6. Stability boundary of the large-amplitude uniform solution $\bar{\psi}_{+}$for $\gamma=1$. The solution exists above the dashed and dotdashed curves. The dashed curve is the saddle-node $h_{s n}(\bar{\eta})$ [Eq. (33)], which is replaced at the point marked with a small square by the dot-dashed curve, indicating the supercritical bifurcation from the zero solution at $h=\sqrt{1+\gamma^{2}}=\sqrt{2}$. The solid curve shows $h_{t h}(\bar{\eta})$ [Eq. (36)] above which $\mathcal{D}<0$. It coincides with the dashed curve at $\bar{\eta}_{c}=-\gamma+\sqrt{1+\gamma^{2}}=\sqrt{2}-1$, indicated by a small circle. In the darkgray region $\bar{\psi}_{+}$is stable because both zeros of the quadratic function in (34) are complex. In the light-gray region $\bar{\psi}_{+}$is stable because both zeros are real and negative. This implies that for $\bar{\eta} \geq \bar{\eta}_{c}$ the large-amplitude solution is always stable, and for $\bar{\eta}<\bar{\eta}_{c}$ the drive $h$ has to exceed the threshold value $h_{t h}(\bar{\eta})$ [Eq. (36)] before the solution becomes stable. Recall that $\bar{\eta}=\eta / 2$.

$$
2 \bar{a}_{ \pm}^{2}=\frac{1-\gamma \bar{\eta} \pm \sqrt{h^{2}\left(1+\bar{\eta}^{2}\right)-(\gamma+\bar{\eta})^{2}}}{1+\bar{\eta}^{2}},
$$

which has to be positive, and

$$
\begin{gathered}
h \cos \left(2 \bar{\theta}_{ \pm}\right)=2 \bar{a}_{ \pm}^{2}-1, \\
h \sin \left(2 \bar{\theta}_{ \pm}\right)=\gamma+\bar{\eta}\left(2 \bar{a}_{ \pm}^{2}\right) .
\end{gathered}
$$

For $\gamma \bar{\eta}<1$ both solutions exist, and a saddle-node bifurcation - obtained by setting the square-root in Eq. (31) to zero-occurs at

$$
h_{s n}(\bar{\eta})=\frac{\gamma+\bar{\eta}}{\sqrt{1+\bar{\eta}^{2}}}, \quad \text { where } \quad \bar{\eta}=\frac{\eta}{2} .
$$

For $\gamma \bar{\eta}>1$ the bifurcation from the zero solution becomes supercritical, occurring at the instability boundary of the zero solution $h=\sqrt{1+\gamma^{2}}$. Note that apart from rescaling $\eta$ by a factor of $3 / 4$ and $a_{+}$by a factor of $\sqrt{2}$ these expressions are identical to those for the approximate amplitude and phase of the soliton solutions (19)-(21).

The modulational instability of the uniform solutions can be evaluated [57] by adding perturbations of the form $\exp ( \pm i k X)$ and calculating their growth rates using the eigenvalues of the matrix $J^{-1} H$, obtained from Eqs. (27) and (28) by substituting $-\partial_{X}^{2}=k^{2}, R=\bar{a}_{ \pm} \cos \bar{\theta}_{ \pm}$, and $I$ $=-a \pm \sin \theta \pm$. The uniform solutions are stable against such modulations as long as the larger of the real parts of the two eigenvalues of $J^{-1} H$ is not positive for any real $k$. This requirement translates to satisfying the inequality 

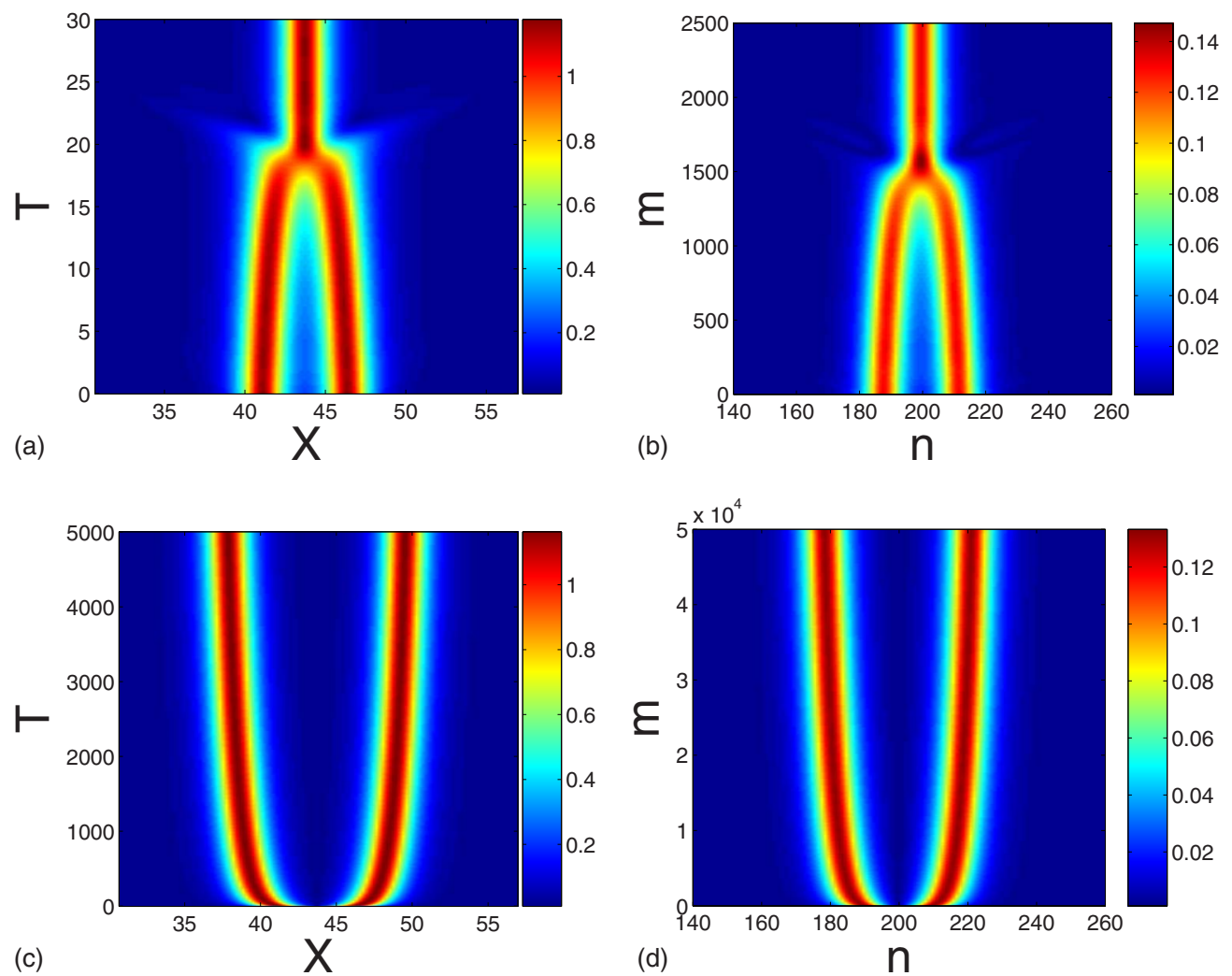

FIG. 7. (Color online) Numerical simulations of soliton interaction. Left and right panels display results obtained, respectively, from numerical simulations of the amplitude equation (8) and of the underlying equations of motion (2), with $h=0.7$ and the remaining parameters as in Fig. 1. (a) and (b): attraction between two in-phase solitons and their merger into a single one, after half the energy has been dissipated. (c) and (d): repulsion between out-of-phase solitons.

$$
s^{2}+2\left(1-4 \bar{a}_{ \pm}^{2}\right) s \pm 8 \bar{a}_{ \pm}^{2} \sqrt{h^{2}\left(1+\bar{\eta}^{2}\right)-(\gamma+\bar{\eta})^{2}} \geq 0
$$

for any non-negative $s=k^{2}$, where a positive sign is assumed for the square root which is real for $h \geq h_{s n}(\bar{\eta})$.

As expected, the small-amplitude uniform solution $\bar{\psi}_{-}$is unstable even against a uniform perturbation, as the left-hand side of Eq. (34) is negative for $k=0$. For the large-amplitude uniform solution $\bar{\psi}_{+}$the inequality (34) is satisfied if either both zeros of the quadratic function of $s$ on the left-hand side are complex, or both are real and nonpositive. The first condition is satisfied if the discriminant

$$
\mathcal{D}=4-32 \bar{\eta} a_{+}^{2}\left(\gamma+2 \bar{\eta} a_{+}^{2}\right)
$$

is negative, and the second condition is satisfied if $\mathcal{D} \geq 0$ and $4 \bar{a}_{+}^{2} \leq 1$ (because the constant term in the quadratic function is positive). Clearly, for $\bar{\eta}=0$ these stability conditions are not satisfied, and the large-amplitude uniform solution is modulationally unstable, in agreement with known results $[57,62]$. However, we indeed find that $\bar{\psi}_{+}$can be stabilized with the help of nonlinear damping, as shown in Fig. 6. If $\bar{\eta} \geq \bar{\eta}_{c}=-\gamma+\sqrt{1+\gamma^{2}}$ then $\bar{\psi}_{+}$is stable everywhere. For weaker nonlinear damping the drive $h$ must exceed a threshold value

$$
\begin{aligned}
h_{t h}(\bar{\eta})= & \frac{1}{2 \bar{\eta}}\left\{1+2 \gamma^{2}\left(1+\bar{\eta}^{2}\right)+4 \gamma \bar{\eta}+5 \bar{\eta}^{2}\right. \\
& \left.-2\left(\gamma+2 \bar{\eta}-\gamma \bar{\eta}^{2}\right) \sqrt{1+\gamma^{2}}\right\}^{1 / 2},
\end{aligned}
$$

determined by substituting the expression for $a_{+}^{2}$ [Eq. (31)] into the discriminant (35), and setting $\mathcal{D}=0$.

If the inequality (34) is not satisfied, the uniform state is modulationally unstable, and the modulation whose growth rate is fastest is expected to appear. This modulation corresponds to the minimum of the quadratic function on the lefthand side of Eq. (34), with wave number $k_{\text {fast }}=\sqrt{4 a_{+}^{2}-1}$.

We demonstrate the use of the stable uniform solution in the dynamical formation of solitons in the bottom panels of Fig. 5, showing a numerical simulation of the equations of motion (2) with periodic boundary conditions, using $N$ $=200$ resonators. The array is initiated with the largeamplitude uniform solution and is driven within the stability boundary of this state. After a long time during which the uniform solution remains stable, the drive amplitude is lowered below the stability threshold (36) for this solution, but within the stability boundaries of the soliton solutions, and solitons are formed via a modulation of the unstable uniform state. The wave number of the modulation that is observed numerically agrees with the predicted value $k_{\text {fast }}$ to within rounding to the nearest mode satisfying the periodic boundary conditions. 


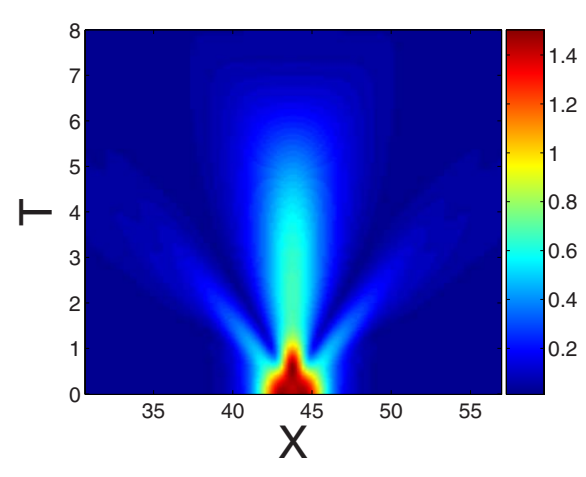

(a) $r_{c}=0.021$

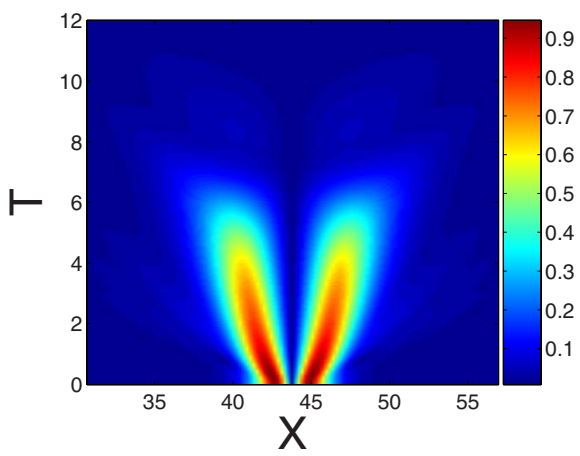

(c) $r_{c}=0.021$

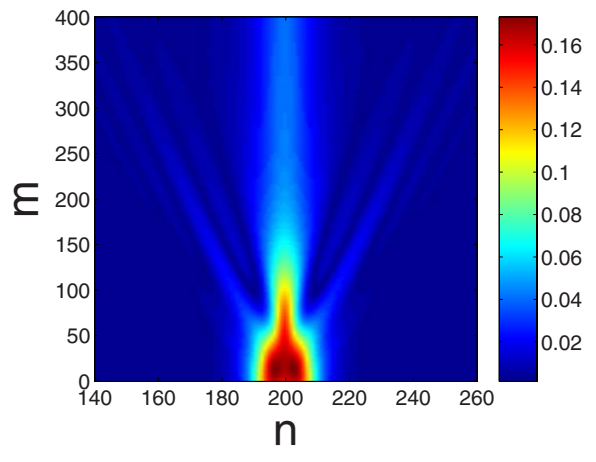

(b) $r_{c}=0.022$

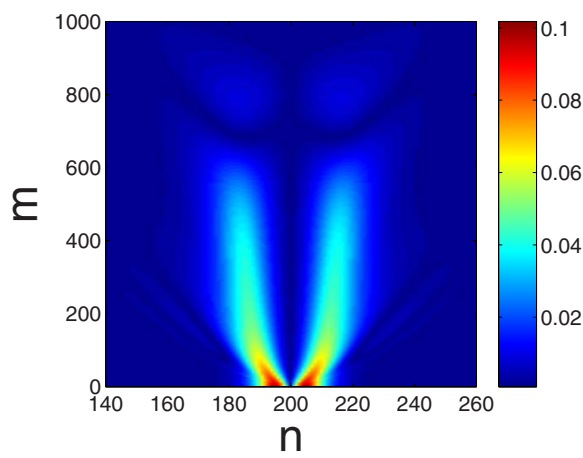

(d) $r_{c}=0.02085$

FIG. 8. (Color online) Annihilation of a pair of strongly overlapping solitons. Left and right panels display results obtained, respectively, from numerical simulations of the amplitude equation (8) and of the underlying equations of motion (2), initiated with a separation given by the indicated values of $r_{c}$ above which annihilation was not observed. (a) and (b): an initial attraction followed by the annihilation of a pair of in-phase solitons. (c) and (d): an initial repulsion followed by the annihilation of a pair of out-of-phase solitons. Parameters are the same as in Fig. 7.

\section{SOLITON INTERACTIONS}

After finding a family of stable soliton solutions of Eq. (8), it is natural to consider the interaction between them. Soliton interactions were studied in detail in the integrable NLS equation, corresponding to $\gamma=h=\eta=0$ in Eq. (7) $[64,65]$. It was found that the interaction of initially stationary solitons depends on their relative phase, with in-phase and out-of-phase solitons attracting and repelling each other, respectively. This property is generic and is not predicated on the integrability of the underlying equations. It is also valid for multidimensional equations [66]. In the presence of additional effects such as amplification and damping the soliton interaction problem is not amenable to a complete analytical study $[67,68]$. However, it is possible to analyze the interaction between two weakly overlapping $\Psi_{+}$solitons by regarding the overlapping nonlinear terms-arising from the substitution of a two-soliton solution into the PDNLS equation-as small perturbations $[49,69,70]$. In this case as well, in-phase solitons attract each other, whereas out-ofphase solitons repel. Using equations derived in Refs. $[67,68,70]$ it is easy to show that adding a small nonlinear damping term does not induce any motion on the solitons, hence one may expect to see the same type of phasedependent interaction in the full amplitude equation (8) with $\eta>0$.
This is indeed verified, as shown in Fig. 7, which presents the results of a numerical integration of the equations of motion (2), and of the amplitude equation (8), simulated as a PDE with initial conditions

$$
\psi_{ \pm}=\psi_{\mathrm{app}}\left(X-(1-r) \frac{L}{2}\right) \pm \psi_{\mathrm{app}}\left(X-(1+r) \frac{L}{2}\right)
$$

where $L=(N+1)(2 \omega \Omega \epsilon / D)^{1 / 2}$ is the scaled length of an array of $N$ resonators, and $r L$ is the distance between the centers of the solitons. Note that one time unit $T=1$ of the amplitude equation is equal to $\omega_{p} /\left[2 \pi\left(\omega_{p}-\omega\right)\right]$ periods of parametric oscillations in the original equations of motion. For the parameters used throughout this paper $\omega_{p} /\left[2 \pi\left(\omega_{p}-\omega\right)\right] \simeq 80$, and one can verify that this is approximately the ratio between the vertical axes of the solutions of the amplitude equation [Figs. 7(a) and 7(c)] and those of the equations of motion [Figs. 7(b) and 7(d)]. Also note that the ratio between the peak heights of the soliton solutions of the amplitude equation and those of the equations of motion in Fig. 7 is approximately $\sqrt{\epsilon}=0.1$, as expected from Eq. (3).

Another effect, which is demonstrated in Fig. 8, is that if the solitons strongly overlap, and $r$ is smaller than some critical distance $r_{c}$, they annihilate into the zero state, for both the in-phase and out-of-phase pairs. For the parameters 


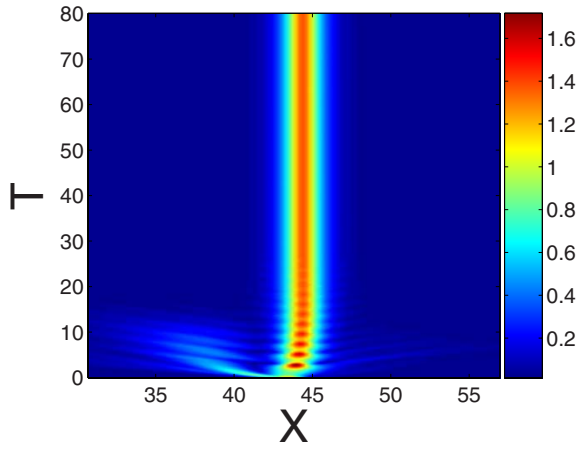

(a) $k=1.35$

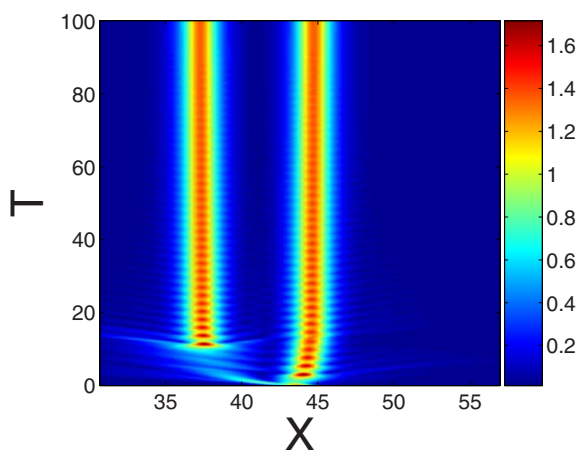

(c) $k=1.37$

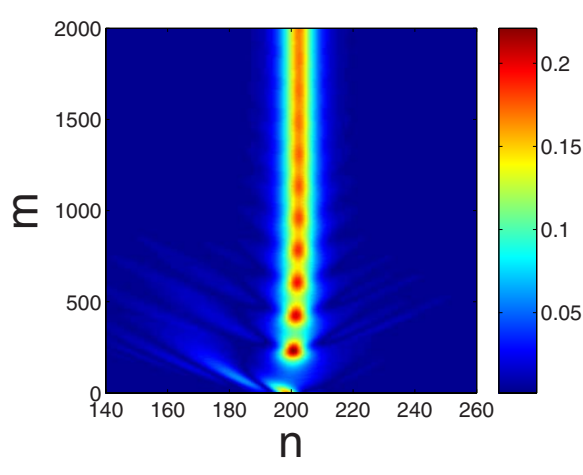

(b) $k=1.35$

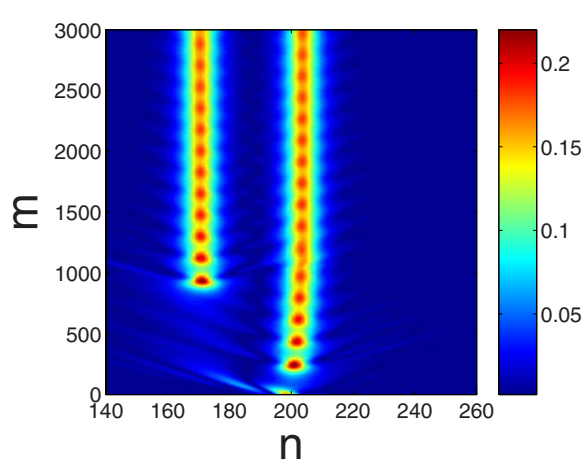

(d) $k=1.37$

FIG. 9. (Color online) Simulations initiated with a boosted soliton. Left and right panels display results obtained, respectively, from numerical simulations of the amplitude equation (8) and of the underlying equations of motion (2), with $\eta=0.02$, $h=1$, and all other parameters as in Fig. 1. Note that the simulations of the equations of motion, displayed on the right, show only the initial stage of the evolution that is simulated with the amplitude equation and displayed on the left (with $T=1$ on the left equivalent to about $m=80$ drive periods on the right, as discussed in the text). (a) and (b): $k=1.35<k_{t h} \simeq 1.36$ and the soliton moves slightly and stops. (c) and (d): $k$ $=1.37>k_{t h}$ and the soliton splits into two. The solutions eventually settle into the known states of one or two stationary solitons.

of Fig. $8, r_{c} \simeq 0.021$. The annihilation of the out-of-phase pair is easily understood because the strongly overlapping solitons cancel each other. However, the mutual destruction of the in-phase pair is a less obvious effect, indicating that for some reason the initial conditions for $r<r_{c}$ are in the basin of attraction of the zero solution, and not in that of the stable single soliton solution, contrary to the case of $r>r_{c}$.

\section{SPLITTING SOLITONS}

The Galilean invariance of the NLS equation admits the motion of any solution at a constant velocity. The parametric drive $h \psi^{*}$ breaks this property of the equation. Nevertheless, stable traveling solitons in the parametrically driven (but undamped) NLS equation were obtained in a numerical form by Barashenkov et al. [71]. We have attempted to do the same with solutions of the full amplitude equation (8) by multiplying the approximate solution $\psi_{\text {app }}$ by $e^{-i k\left(X-X_{0}\right)}$, thereby boosting it. We have concluded that such a boost may set the soliton into transient motion, but eventually it comes to a complete halt, as shown in Figs. 9(a) and 9(b).

For certain parameter values we observe a noteworthy effect in which a boosted soliton splits into two. In order to estimate the threshold value $k_{t h}$ for the wave number $k$, above which this splitting occurs, we write the energy of the soliton using the Hamiltonian density that gives rise to the driven, but undamped, NLS equation,

$$
E=\int_{-\infty}^{\infty}\left(\left|\frac{\partial \psi}{\partial X}\right|^{2}+|\psi|^{2}-|\psi|^{4}+h \operatorname{Re}\left(\psi^{2}\right)\right) d X
$$

Following Eq. (8), this energy evolves in time according to

$$
\begin{aligned}
\frac{d E}{d T}= & 2 \int_{-\infty}^{\infty}\left(\gamma+\eta|\psi|^{2}\right)\left(\frac{1}{2}\left(\psi \frac{\partial^{2} \psi^{*}}{\partial X^{2}}+\psi^{*} \frac{\partial^{2} \psi}{\partial X^{2}}\right)\right. \\
& \left.-|\psi|^{2}+2|\psi|^{4}-h \operatorname{Re}\left(\psi^{2}\right)\right) d X
\end{aligned}
$$

The right-hand side of Eq. (39) is zero for $\psi$ $=a e^{-i \theta} \operatorname{sech}(a X)$ and $h \cos (2 \theta)=a^{2}-1$ for any constant $a$-in particular, for the approximate solution $\psi_{\text {app }}$ as well as for the numerically exact solution. By substituting the boosted approximate solution $\psi_{\text {app }}(X) e^{-i k\left(X-X_{0}\right)}$ into Eq. (38), we find its energy to be 


$$
E(k)=2 a_{+}\left(1+k^{2}\right)+\frac{2 k \pi\left(a_{+}^{2}-1\right)}{\sinh \left(k \pi / a_{+}\right)}-\frac{2}{3} a_{+}^{3},
$$

whereas for the static soliton $E(k=0)=4 a_{+}^{3} / 3$. Thus, an obvious estimate for the threshold wave number $k_{t h}$ required to split a soliton into two is given by the condition $E\left(k_{t h}\right)$ $=2 E(0)$. For the parameters of Fig. $9, k_{t h} \simeq 1.36$, and indeed below this value the soliton does not split [in (a) and (b) $k$ $=1.35]$ while above this value the soliton does split [in (c) and (d) $k=1.37$ ]. At still larger values of $k$ the soliton is destroyed by the boost and eventually decays to zero. For the parameters of Fig. 9 this happens for $k>1.59$. We note that although it might seem plausible to have values of $k$ for which boosting a single soliton would split it into three, we were unable to detect such an effect.

\section{BOUND STATES}

We have considered the effects of pairwise interaction between solitons, and of boosting a single static soliton. It was shown by Barashenkov and Zemlyanaya [59] that a combination of both features within the framework of the PDNLS equation may lead to the formation of solitonic complexes, or bound states [68]. These complexes were found numerically, solving the PDNLS with an initial guess of the form

$$
\psi_{\mathrm{b}}=\psi\left(X-X_{0}\right) e^{i k\left(X-X_{0}\right)}+\psi\left(X+X_{0}\right) e^{-i k\left(X+X_{0}\right)},
$$

where $\psi(X)=A \operatorname{sech}(A X) e^{-i \theta}$. For $\gamma=0.565$ and $h=0.9$ the rest of the parameters were found by means of a variational procedure elaborated in [59] to be $\theta=\Theta_{+}, A=1.14$, $k=-0.068$, and $X_{0}=2.017$.

Using the PDNLS variational ansatz (41) as an initial guess, we are able to obtain stationary solitonic bound states for the full amplitude equation (8) with $\eta>0$. Performing a linear stability analysis on these solutions, as described earlier using Eq. (29), reveals that some of them are stable. Figure 10 shows one of these stable bound state solutions, obtained numerically using the amplitude equation (8), and nicely reproduced by a numerical integration of the underlying equations of motion (2).

\section{CONCLUSIONS}

We have investigated intrinsic localization of vibration in response to parametric excitation in an array of resonators with a stiffening nonlinearity. Our analysis was chiefly performed on a single amplitude equation, which was derived directly from the underlying equations of motion of the array to describe the slow spatiotemporal dynamics of the system. The discreteness of the array imposes an upper bound on the spectrum of linear modes. We have studied the case in which neighboring resonators oscillate out-of-phase, in the staggered mode, with an oscillation frequency set slightly above the top frequency of the linear spectrum. One can similarly study ILMs in resonators with a softening nonlinearity, by changing the sign of $u_{n}^{3}$ in the equations of motion (2), and considering the case in which neighboring resonators oscillate in-phase with an oscillation frequency set slightly below the bottom frequency of the linear spectrum.

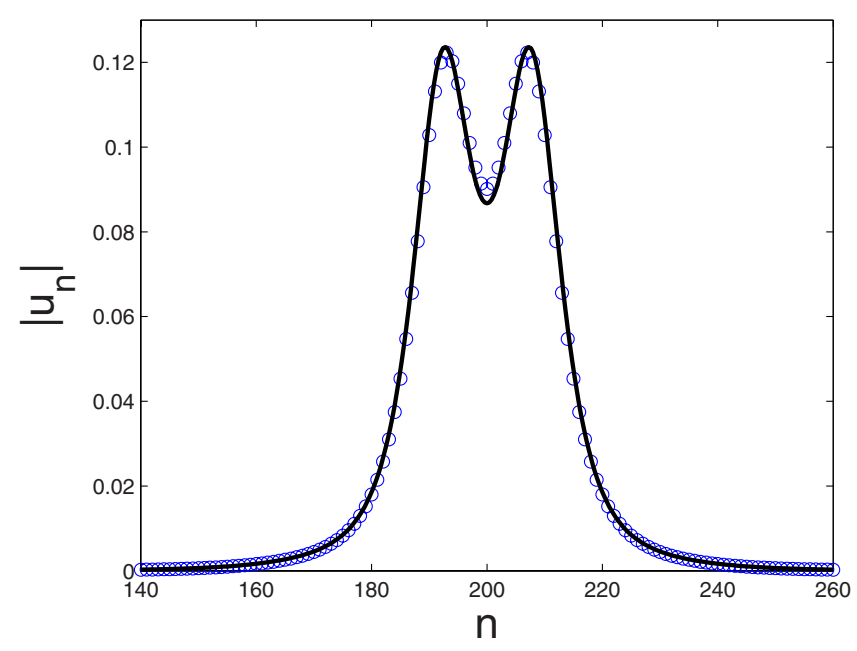

FIG. 10. (Color online) A stable bound state of two solitons. The os are absolute values of the displacements of the resonators, obtained by a numerical integration of the discrete equations of motion (2), after a sufficiently long transient time has elapsed. The solid line is the stable solution obtained by solving the amplitude equation (8) as a boundary value problem. The parameters are $\gamma=0.565, h=1, \eta=0.3$, and all others as in Fig. 1 .

The array that we consider, hence also the amplitude equation we derive, are nonlinearly damped. Its localized modes emerge from two exact soliton solutions that exist in the absence of nonlinear damping. We have shown that nonlinear damping increases the range of parameters for which localized solutions are stable. However, nonlinear damping increases the region in which the zero state is the only stable one, and it also stabilizes the nonzero uniform solution of the amplitude equation, which is modulationally unstable if $\eta$ $=0$. We have studied soliton interaction and soliton splitting, both in the presence of nonlinear damping. We have also found a family of localized solutions in the form of bound states of two solitons. In a follow-up work, we intend to perform a more detailed investigation of the different localized solutions of the full amplitude equation (8) with $\eta>0$, using numerical continuation.

All results obtained from the amplitude equation are in excellent agreement with numerical solutions of the underlying equations of motion. This upholds the validity of using a continuous PDE as a tool for analyzing ILMs, or discrete solitons, in a system whose original description is given in terms of coupled ordinary differential equations. Furthermore, our numerical simulations of the equations of motion suggest that the predicted effects can be observed in parametrically driven arrays of real MEMS and NEMS resonators, thus motivating new experiments in these systems.

\section{ACKNOWLEDGMENTS}

We thank an anonymous referee for inquiring about the dynamical formation of solitons in our array, which prompted us to add Sec. V. This work was supported by the U.S.-Israel Binational Science Foundation (BSF) through Grant No. 2004339, and by the Israeli Ministry of Science and Technology. 
[1] R. Lifshitz and M. C. Cross, in Review of Nonlinear Dynamics and Complexity, edited by H. G. Schuster (Wiley, Weinheim, 2008), Vol. 1, pp. 1-52.

[2] K. L. Turner, S. A. Miller, P. G. Hartwell, N. C. MacDonald, S. H. Strogatz, and S. G. Adams, Nature (London) 396, 149 (1998).

[3] H. G. Craighead, Science 290, 1532 (2000).

[4] E. Buks and M. L. Roukes, Europhys. Lett. 54, 220 (2001).

[5] D. V. Scheible, A. Erbe, R. H. Blick, and G. Corso, Appl. Phys. Lett. 81, 1884 (2002).

[6] W. Zhang, R. Baskaran, and K. L. Turner, Sens. Actuators, A 102, 139 (2002).

[7] W. Zhang, R. Baskaran, and K. Turner, Appl. Phys. Lett. 82, 130 (2003).

[8] M.-F. Yu, G. J. Wagner, R. S. Ruoff, and M. J. Dyer, Phys. Rev. B 66, 073406 (2002).

[9] J. S. Aldridge and A. N. Cleland, Phys. Rev. Lett. 94, 156403 (2005).

[10] A. Erbe, H. Krommer, A. Kraus, R. H. Blick, G. Corso, and K. Richter, Appl. Phys. Lett. 77, 3102 (2000).

[11] I. Kozinsky, H. W. C. Postma, O. Kogan, A. Husain, and M. L. Roukes, Phys. Rev. Lett. 99, 207201 (2007).

[12] B. DeMartini, J. Rhoads, K. Turner, S. Shaw, and J. Moehlis, J. Microelectromech. Syst. 16, 310 (2007).

[13] S. C. Masmanidis, R. B. Karabalin, I. De Vlaminck, G. Borghs, M. R. Freeman, and M. L. Roukes, Science 317, 780 (2007).

[14] I. Katz, A. Retzker, R. Straub, and R. Lifshitz, Phys. Rev. Lett. 99, 040404 (2007).

[15] I. Katz, R. Lifshitz, A. Retzker, and R. Straub, New J. Phys. 10, 125023 (2008).

[16] X. M. H. Huang, C. A. Zorman, M. Mehregany, and M. L. Roukes, Nature (London) 421, 496 (2003).

[17] A. N. Cleland and M. R. Geller, Phys. Rev. Lett. 93, 070501 (2004).

[18] E. Buks and M. L. Roukes, J. Microelectromech. Syst. 11, 802 (2002).

[19] R. Lifshitz and M. C. Cross, Phys. Rev. B 67, 134302 (2003).

[20] Y. Bromberg, M. C. Cross, and R. Lifshitz, Phys. Rev. E 73, 016214 (2006).

[21] E. Kenig, R. Lifshitz, and M. C. Cross, Phys. Rev. E 79, 026203 (2009).

[22] M. C. Cross, A. Zumdieck, R. Lifshitz, and J. L. Rogers, Phys. Rev. Lett. 93, 224101 (2004).

[23] M. C. Cross, J. L. Rogers, R. Lifshitz, and A. Zumdieck, Phys. Rev. E 73, 036205 (2006).

[24] A. J. Sievers and S. Takeno, Phys. Rev. Lett. 61, 970 (1988).

[25] D. K. Campbell, S. Flach, and Y. S. Kivshar, Phys. Today 57, 43 (2004).

[26] P. Maniadis and S. Flach, Europhys. Lett. 74, 452 (2006).

[27] M. Sato, B. E. Hubbard, L. Q. English, A. J. Sievers, B. Ilic, D. A. Czaplewski, and H. G. Craighead, Chaos 13, 702 (2003).

[28] M. Sato, B. E. Hubbard, A. J. Sievers, B. Ilic, D. A. Czaplewski, and H. G. Craighead, Phys. Rev. Lett. 90, 044102 (2003).

[29] M. Sato, B. E. Hubbard, A. J. Sievers, B. Ilic, and H. G. Craighead, Europhys. Lett. 66, 318 (2004).

[30] M. Sato, B. E. Hubbard, and A. J. Sievers, Rev. Mod. Phys. 78, 137 (2006).
[31] M. Sato and A. J. Sievers, Phys. Rev. Lett. 98, 214101 (2007).

[32] M. Sato and A. J. Sievers, Low Temp. Phys. 34, 543 (2008).

[33] E. Trías, J. J. Mazo, and T. P. Orlando, Phys. Rev. Lett. 84, 741 (2000).

[34] P. Binder, D. Abraimov, A. V. Ustinov, S. Flach, and Y. Zolotaryuk, Phys. Rev. Lett. 84, 745 (2000).

[35] H. S. Eisenberg, Y. Silberberg, R. Morandotti, A. R. Boyd, and J. S. Aitchison, Phys. Rev. Lett. 81, 3383 (1998).

[36] H. S. Eisenberg, R. Morandotti, Y. Silberberg, S. Bar-Ad, D. Ross, and J. S. Aitchison, Phys. Rev. Lett. 87, 043902 (2001).

[37] D. Cheskis, S. Bar-Ad, R. Morandotti, J. S. Aitchison, H. S. Eisenberg, Y. Silberberg, and D. Ross, Phys. Rev. Lett. 91, 223901 (2003).

[38] J. W. Fleischer, M. Segev, N. K. Efremidis, and D. N. Christodoulides, Nature (London) 422, 147 (2003).

[39] B. I. Swanson, J. A. Brozik, S. P. Love, G. F. Strouse, A. P. Shreve, A. R. Bishop, W.-Z. Wang, and M. I. Salkola, Phys. Rev. Lett. 82, 3288 (1999).

[40] U. T. Schwarz, L. Q. English, and A. J. Sievers, Phys. Rev. Lett. 83, 223 (1999).

[41] M. Sato and A. J. Sievers, Nature (London) 432, 486 (2004).

[42] S. Zaitsev, R. Almog, O. Shtempluck, and E. Buks, in Proccedings of the 2005 International Conference on MEMS, NANO, and Smart Systems (ICMENS 2005) (IEEE Computer Society, Los Alamitos, CA, 2005), pp. 387-391.

[43] L. D. Landau and E. M. Lifshitz, Mechanics, 3rd ed. (Butterworth-Heinemann, Oxford, 1976), Sec. 27.

[44] M. C. Cross and P. C. Hohenberg, Rev. Mod. Phys. 65, 851 (1993).

[45] W. Zhang and J. Viñals, Phys. Rev. Lett. 74, 690 (1995).

[46] X. Wang and R. Wei, Phys. Rev. Lett. 78, 2744 (1997).

[47] X. Wang and R. Wei, Phys. Rev. E 57, 2405 (1998).

[48] G. Miao and R. Wei, Phys. Rev. E 59, 4075 (1999).

[49] S. Longhi, Phys. Rev. E 53, 5520 (1996).

[50] V. J. Sánchez-Morcillo, I. Pérez-Arjona, F. Silva, G. J. de Valcárcel, and E. Roldán, Opt. Lett. 25, 957 (2000).

[51] B. Denardo, B. Galvin, A. Greenfield, A. Larraza, S. Putterman, and W. Wright, Phys. Rev. Lett. 68, 1730 (1992).

[52] W.-Z. Chen, Phys. Rev. B 49, 15063 (1994).

[53] N. V. Alexeeva, I. V. Barashenkov, and G. P. Tsironis, Phys. Rev. Lett. 84, 3053 (2000).

[54] N. Dror and B. A. Malomed, Phys. Rev. E 79, 016605 (2009).

[55] J. Burke, A. Yochelis, and E. Knobloch, SIAM J. Appl. Dyn. Syst. 7, 651 (2008).

[56] I. V. Barashenkov, M. M. Bogdan, and V. Korobov, Europhys. Lett. 15, 113 (1991).

[57] I. V. Barashenkov, S. Cross, and B. A. Malomed, Phys. Rev. E 68, 056605 (2003).

[58] E. V. Zemlyanaya and I. V. Barashenkov, SIAM J. Appl. Math. 64, 800 (2004).

[59] I. V. Barashenkov and E. V. Zemlyanaya, Phys. Rev. Lett. 83, 2568 (1999).

[60] I. V. Barashenkov and Y. S. Smirnov, Phys. Rev. E 54, 5707 (1996).

[61] M. Bondila, I. V. Barashenkov, and M. M. Bogdan, Physica D 87, 314 (1995).

[62] X. Wang, Physica D 154, 337 (2001).

[63] R. B. Thakur, L. Q. English, and A. J. Sievers, J. Phys. D 41, 015503 (2008). 
[64] J. P. Gordon, Opt. Lett. 8, 596 (1983).

[65] C. Desem and P. Chu, Opt. Lett. 12, 349 (1987).

[66] B. A. Malomed, Phys. Rev. E 58, 7928 (1998).

[67] V. V. Afanasjev, B. A. Malomed, and P. L. Chu, Phys. Rev. E 56, 6020 (1997).
[68] B. A. Malomed, Phys. Rev. A 44, 6954 (1991).

[69] V. I. Karpman and V. V. Solov'ev, Physica D 3, 487 (1981).

[70] S. Longhi, Phys. Rev. E 55, 1060 (1997).

[71] I. V. Barashenkov, E. V. Zemlyanaya, and M. Bär, Phys. Rev. E 64, 016603 (2001). 